\title{
CDISC Protocol Entities Protocol Contact Role Response Terminology
}

National Cancer Institute

\section{Source}

National Cancer Institute. CDISC Protocol Entities Protocol Contact Role Response

Terminology. NCI Thesaurus. Code C154681.

Terminology associated with the protocol contact role response codelist of the Clinical Data Interchange Standards Consortium (CDISC) protocol entities. 